
\title{
25 Research Soure \\ Excavation of a Novel Transcription Factors-related Prognostic Signature for Osteosarcoma
}

\section{Long Cheng}

Jilin University First Hospital

Yi Liu

Jilin University First Hospital

Xu Huang

Jilin University First Hospital

Jiting Zhang

Jilin University First Hospital

Bo Wu

Jilin University First Hospital

Yanwei Liu

Jilin University First Hospital

Zhaoyu Fu

Jilin University First Hospital

Dong Zhu ( $\square$ zhu_dong@mail.jlu.edu.cn )

Jilin University First Hospital

\section{Research}

Keywords: Osteosarcoma, transcription factors-related prognostic signature, prognosis, nomogram, the Therapeutically Applicable Research to Generate Effective Treatments database

Posted Date: September 13th, 2021

DOl: https://doi.org/10.21203/rs.3.rs-847325/v1

License: (c) (1) This work is licensed under a Creative Commons Attribution 4.0 International License.

Read Full License 


\section{Abstract}

\section{Background}

Transcription factors (TFs) are involved in the initiation and development of many cancers, regulating cancer-related activities. However, the significance of TF-related genes in predicting the prognosis of osteosarcoma (OS) patients is not yet clear. Risk stratification using prognostic markers can facilitate clinical decision-making and effect in the treatment of cancer.

Material and methods

In the study, we aimed to establish an optimal TF signature for predicting the prognosis of OS patients. We identified 24 differentially expressed TFs in metastatic and non-metastatic OS samples from the Therapeutically Applicable Research to Generate Effective Treatments (TARGET) database. Subsequently, we performed univariate and multivariate cox regression analysis to built a TFs-related prognostic signature (TRPS) confirmed in an independent cohort (GSE39055). The ESTIMATE algorithm was used to estimate the immune/stromal cell score.

Results

We built a TRPS for OS patients, including MESP1 and ZNF597. Kaplan-Meier (KM) survival analysis and receiver operating characteristic (ROC) curve both confirmed the accuracy of the signature. Multivariate analysis proved that this TRPS was an independent prognostic predictor of OS, and it was further confirmed in the GSE39055 dataset and multiple clinical subtypes. In addition, we found a significant negative correlation between stromal score and risk score. Moreover, the relative abundance of NK cells in the low-risk prognosis group was notably higher than that in the high-risk prognosis group.

\section{Conclusion}

In summary, we established a TFs-related prognostic signature with high diagnostic, prognostic efficacy in OS patients, which may optimize the prognostic management of osteosarcoma patients and help achieve individualized treatment.

\section{Introduction}

Osteosarcoma (OS) is one of the most common primary bone malignant tumors affecting children, adolescents, and young adults[1]. OS primarily came from malignant bone mesenchymal cells[2]. Currently, the primary therapies include operation, chemotherapy, targeted therapy, and immune therapy, the outcomes of OS patients remain not very ideal[3,4]. The 5 -year overall survival is only about $20 \%$ once it develops metastatic disease[5]. Therefore it is important to explore novel prognostic biomarkers and optimize personalized management of OS patients. Numerous studies have shown that molecular risk assignments could facilitate prognostic assessment efficiently and guide individualized precision therapy of OS patients[6-8]. 
Transcription factors (TFs) can identify specific DNA sequences to regulate transcription[9]. Many studies have found that TFs are often involved in the occurrence, development, metastasis and migration of many human cancers, including OS[10,11]. For example, Runx2 is thought to be related to metastasis and poor response to chemotherapy in OS patients $[12,13]$. The stem cell transcription factor Sox 2 is considered a key factor in the development of OS and the proliferation of cancer cells[14]. Overexpression of EMT transcription factor (EMT-TF)[15] and Signal transducer and activator of transcription 3 (STAT3) is correlated with poor outcome of OS[16]. These findings indicate that TFs play an essential role in the development and prognosis of OS. To this end, we screened differentially expressed TFs (DETFs) in metastatic and non-metastatic OS samples. We established a TFs-related prognostic signature (TRPS) that significantly correlated with the prognosis of OS patients and validated it in another independent cohort. In conclusion, our study discovers a novel TRPS, a promising independent prognostic biomarker of OS.

\section{Materials And Methods \\ 2.1. Data collection}

We gathered the mRNA expression data and the corresponding clinical data of OS patients from the TARGET database (https://ocg.cancer.gov/programs/target/). The mRNA expression data and clinical follow-up data of OS samples in GSE39055 are downloaded from the GEO database (https:// www. ncbi. $\mathrm{nlm}$. nih. gov/geo/). The clinical information of the OS samples in the two data sets is shown in Supplementary Table 1. And the TF list was gathered from the Human Transcription Factors website (http://humantfs.ccbr.utoronto.ca/).[17] The workflow chart of this study is in Supplementary Fig. 1.

\subsection{Screening of differentially expressed transcription factors}

According to the mRNA expression profiles and clinical follow-up information of OS samples in the TARGET data set, we divided the patients into metastatic and non-metastatic groups and screened TFrelated mRNAs. Using the $\mathrm{R}$ package limma and Wilcox. test in RStudio software, we ascertained DETFs between the two groups. $|\log (\mathrm{FC})| \geq 0.5$ and $p$-value $<0.05$ were set as the cut-off value.

\subsection{Construction and assessment of the TFs-related prognostic signature}

We performed univariate analysis to ascertain independent prognostic TFs. Then, the TRPS was established on the bias of the multivariate Cox stepwise regression analysis results. We calculated the risk score of each patient based on the expression level of DETFs and the coefficient calculated by the multivariate Cox regression model. Finally, according to the optimal cut-off value determined by the X-tile 3.6.1 software, OS patients were divided into low and high-risk groups. The Kaplan-Meier survival analysis and the ROC curve was used to validate the accuracy of the TRPS. Next, we divided the OS patients in GSE39055 datasets into low- and high-risk groups on the bias of the same formula, verifying 
the applicability and utility of the TRPS. We used univariate and multivariate cox regression to ascertain the independence of risk signature in predicting the prognosis of OS patients. According to age, gender, site, metastatic, and risk score, we built a nomogram through the R package "rms".

\subsection{The correlation between the TFs-related prognostic signature and the immune microenvironment}

According to the mRNA expression data, we used the ESTIMATE algorithm [18] to calculate the immune score and stromal score of each OS sample. Then, based on the median value of immune and stromal scores, the patients were divided into high scores and low scores groups. We evaluated the relative abundance of 28 subpopulations in the TARGET cohort by single-sample gene set enrichment analysis (ssGSEA), including Treg, Tfh, TAM, macrophages, neutrophils, T helper cells, etc.[19].

\section{Results}

\subsection{Identification of differentially expressed transcription factors related to OS patients}

In the TARGET dataset, compared with non-metastatic primary samples $(n=65)$, we screened 24 DETFs in metastatic osteosarcoma samples $(n=22)$. Among them, 23 genes were down-regulated in metastatic tumor tissues, and 1 gene was up-regulated in metastatic tumor tissues (Fig. 1A, Fig. 1B).

\subsection{Construction and assessment of the multi-TF predictive model}

We carried on univariate regression analysis to establish TFs-related gene signature in osteosarcoma. And three genes are ascertained to be obviously correlated with the outcome of OS patients.

(Supplementary Table 2, Fig. 1C). Then, based on multivariate cox regression analysis, we established a prognostic risk model on the bias of two genes (ZNF597 and MESP1). The risk score of each OS patient was calculated as follows: [-1.4218* ZNF597] + [0.4236* MESP1]. Then we split the OS samples into high and low-risk groups based on the optimal cut-off value of the risk score calculated by the X-Tile software (2.24) (Supplement Fig. 2). Then based on the TRPS, we plotted the expression heat map, the risk score distribution and the survival status between the two risk groups (Fig. 1D). The Kaplan-Meier survival curve suggested that the prognosis of OS patients in the low-risk group was observably better than that in the high-risk group (Fig. 1E). ROC curve results suggested that the AUCs were $0.870,0.868$, and 0.777 at 1, 2, and 3 years, which indicated our TRPS had a high predictive performance (Fig. 1F). And as shown in Supplement Fig. 3, the AUCs for 1-, 3- and 5-year OS predictions of the TRPS were higher than AUCs of age, gender, and site in the TARGET cohort. And we found that the metastatic OS samples showed a higher risk score than the non-metastatic samples $(P<0.001)$, indicating that the signature is closely correlated with the metastasis. (Fig. 1G). 


\subsection{The verification of the TFs-related prognostic signature in an independent cohort}

We did a validation analysis in another independent cohort (GSE39055) to ascertain whether the TRPS has a better predictive value for OS patient survival. The patients were split into low- and high-risk prognostic groups using the same formula and cut-off value (2.24). The Kaplan-Meier survival analysis suggested that the prognosis of OS patients in the high-risk group was markedly worse than that in the low-risk group (Fig. 2A). And the ROC curve results showed that the 3-year predicted AUC was 0.759 (Fig. 2B). Similarly, independent predictive analysis suggests that risk score can also be regarded as an independent prognostic factor in the GSE39055 data set (Fig. 2C, D). These results demonstrated that our TRPS could also have good reproducibility in other independent cohorts of OS patients.

\subsection{Independence predictive activity of the TFs-related prognostic signature in OS patients}

As we all know, a promising prognostic biomarker is supposed to be independent of other clinicopathological factors to predict patient survival, such as age, sex, and metastasis. Therefore we regrouped the OS patients on the basis of different clinical characteristics and conducted Kaplan-Meier survival curves, respectively. The results manifested that the outcome of OS patients in the low-risk group was observably better than that in the high-risk group regardless of age, gender, and metastasis $(p<0.05$, Fig. 2E, F, and G). Also, we conducted the univariate and multivariate prognostic analysis indicating that our TRPS is an independent predictor. (Fig. 3A, B). All of the results manifested that the TRPS had satisfactory independence and applicability in OS patients. In addition, we constructed the nomogram for predicting 1-,3-, and 5- year survival rates on the bias of the risk score and other clinicopathological factors (Fig. 3C).

\subsection{The correlation between the TFs-related prognostic signature and the immune microenvironment}

We use the ESTIMATE algorithm to estimate the immune cell and stromal cell scores of the OS samples in the TARGET database. We found that the overall survival rate of OS samples with the lower stromal score was lower than that of the high stromal score group (Fig. 4A). Moreover, the stromal scores of patients in the high-risk group were obviously lower than those in the low-risk group (Fig. 4B). Therefore, we speculate that the risk score and stromal scores are negatively correlated (Fig. 4C). As for immune cell infiltration (ICI), we carried on SSGSEA analysis on the OS samples in the TARGET cohort (Fig. 4D, E) and GSE39055 data set (Fig. 4F, G). The intersection of the two data sets showed that the abundance of NK cells in the low-risk prognosis group was notably higher than that in the high-risk prognosis group. And we found that the abundance of NK cells also had a difference between the metastatic and the nonmetastatic OS groups (Fig. 4H). The abundance of NK cells in the metastatic group was notably lower than that in the non-metastatic group. 


\section{Discussion}

Osteosarcoma is the most frequent primary solid malignancy of bone, and the most common sites of occurrence are the metaphysis of long bones[20,21]. The 5-year overall survival is meagre once it develops the metastatic disease. Transcription factors play an essential part in regulating gene expression, while several stages involved in tumor formation require modifications of gene expression programs[22]. Recently, a large number of TFs- related prognostic genes have been discovered in many cancers. These genes are combined to establish TFs-related prognostic signatures, such as liver cancer[23] and head and neck squamous cell carcinoma[24]. However, the TFs-related prognostic signature has not yet been established in OS. Therefore, we screened DETFs from osteosarcoma samples in the TARGET database and established a TRPS to predict the prognosis accurately.

Our study ascertained 24 DETFs in non-metastatic and metastatic OS samples. Then we used univariate and multivariate Cox regression analysis to construct a TRPS with high diagnostic, prognostic efficacy in OS patients (MESP1, ZNF597). Post Mesoderm 1 (MESP1), as a basic helix-loop-helix transcription factor member, is the primary regulatory gene for mesendoderm formation[25]. MESP1 is considered to be the primary regulator of the cardiovascular lineage[26]. MESP1 expression is elevated in many cancers. MESP1 has been reported to be regarded as an oncogene in lung cancer[27]. And research has reported that MESP1 can regulate skeletal muscle differentiation in a context-sensitive manner without serumderived factors [28]. Therefore, we suggested that MESP1 might be involved in the development of the OS. ZNF597 belongs to a human specifically imprinted gene encoding zinc finger protein[29]. There are few previous studies on ZNF597, and the methylation of ZNF597 is negatively correlated with ambient temperature[30]. Studies have shown that the hypomethylation of ZNF597 is correlated with some rare diseases, such as imprinting diseases[31], brain development disorders[32] and silver-Russell syndrome[33]. At present, we know very little about ZNF597, and its biological functions need to be further explored.

We next assessed the accuracy of the TRPS by conducting the Kaplan-Meier analyses and ROC analyses and did a validation analysis in another independent cohort (GSE39055). Moreover, clinical subgroup, univariate and multivariate Cox regression analyses both showed the independent predictive activity of the signature in OS patients. All the results indicated that the signature was a powerful predictor prognosis of OS.

Moreover, we studied the correlation between the immune/stromal cells infiltrated in tumor microenvironment (TME) and the prognostic signature. We found that the score of infiltrated stromal cells in TME is closely correlated with OS prognosis and notably negatively correlated with the risk score. These results indicated that we could predict the relative content of stromal cells in the TME of OS patients based on the risk score.

Natural killer (NK) cells are lymphocytes with cytotoxic activity that can target and kill malignant cells[34]. Activated NK cells can release cytokines and induce cancer cell lysis through various mechanisms, playing an essential role in controlling tumor progression and metastasis[35, 36]. Research has pointed 
out that NK cell activators have achieved a good prognosis in treating high-grade osteosarcoma[37]. And a recent study indicated that the relative abundance of NK cells in the non-recurrent OS group was higher than that in the recurrent group[38]. Our results indicated that the low-risk prognosis group had a higher abundance of NK cells than the high-risk prognosis group. Moreover, we constructed a nomogram for effectively predicting 1-, 3-, and 5-year survival rates on the bias of the risk score and other clinicopathological factors.

Although we provide promising prognostic biomarkers for OS with high clinical value, this study also has some unavoidable limitations, as with all research. First, the sample size for osteosarcoma in the public database was small, and some clinical information was incomplete, which may influence our subgroup analysis. Secondly, there is no functional experiment to verify the specific mechanism of MESP1 and ZNF597 in osteosarcoma. Therefore, it is important to design prospective studies to verify our findings, covering more extensive and comprehensive samples.

\section{Conclusion}

In summary, we established a TFs-related prognostic signature with high diagnostic, prognostic efficacy in OS patients (MESP1 and ZNF597) and did a further validation analysis in another independent cohort (GSE39055). The signature can conduct risk stratification focusing on OS patients and facilitate personalized management. Clinical subgroup, univariate and multivariate Cox regression analyses showed the independent predictive activity of the signature in OS patients. In addition, we found that the risk signature is closely related to TME. The TFs-related prognostic signature can be used as a prognostic biomarker of OS patients, stimulating new ideas for TF-targeted therapy and promote personalized clinical treatment. However, more research is needed to verify our findings.

\section{Abbreviations}

TF: Transcription factor;

OS: osteosarcoma;

TRPS: transcription factors-related prognostic signature

DETFs: differentially expressed transcription factors

TARGET: Therapeutically Applicable Research to Generate Effective Treatments

ROC: receiver operating characteristic;

ssGSEA: single-sample gene set enrichment analysis

ICl: immune cell infiltration 
ZNF597: zinc finger protein 597

MESP1: mesoderm posterior bHLH transcription factor 1

\section{Declarations}

\section{Ethics approval and consent to participate}

Not applicable.

\section{Consent for publication}

Not applicable.

\section{Availability of data and materials}

The datasets used and/or analyzed during the current study are available from the corresponding author upon reasonable request.

\section{Competing interests}

The authors declare that they have no competing interests.

\section{Funding}

This study was supported by the National Natural Science Foundation of China (Grant No12072129).

\section{Authors' contributions}

Long Cheng and Yi Liu contributed to the conception of the work. Yi Liu, Dong Zhu, and Xu Huang searched the literature and extracted the data. Long Cheng and Dong Zhu wrote the manuscript. Jiting Zhang, Bo Wu, Yanwei Liu, and Zhaoyu Fu revised the manuscript. All authors read and approved the manuscript.

\section{Acknowledgements}

We thank Dong Zhu for his advice and support on bioinformatics analysis.

\section{References}

1.L.A. Doyle, Sarcoma classification: an update based on the 2013 World Health Organization Classification of Tumors of Soft Tissue and Bone, Cancer 120 (2014) 1763-1774. 10.1002/cncr.28657.

2.A.J. Saraf, J.M. Fenger, R.D. Roberts, Osteosarcoma: Accelerating Progress Makes for a Hopeful Future, Front Oncol 8 (2018) 4. 10.3389/fonc.2018.00004. 
3.A. Luetke, P.A. Meyers, I. Lewis, H. Juergens, Osteosarcoma treatment - where do we stand? A state of the art review, Cancer Treat Rev 40 (2014) 523-532. 10.1016/j.ctrv.2013.11.006.

4.G.M. Shankar, M.J. Clarke, T. Ailon, L.D. Rhines, S.R. Patel, A. Sahgal, I. Laufer, D. Chou, M.H. Bilsky, D.M. Sciubba, M.G. Fehlings, C.G. Fisher, Z.L. Gokaslan, J.H. Shin, The role of revision surgery and adjuvant therapy following subtotal resection of osteosarcoma of the spine: a systematic review with metaanalysis, J Neurosurg Spine 27 (2017). 10.3171/2016.12.SPINE16995.

5.M. Zhang, X. Zhang, Association of MMP-2 expression and prognosis in osteosarcoma patients, Int J Clin Exp Pathol 8 (2015) 14965-14970.

6.R.J. Flores, A.J. Kelly, Y. Li, M. Nakka, D.A. Barkauskas, M. Krailo, L.L. Wang, L. Perlaky, C.C. Lau, M.J. Hicks, T.-K. Man, A novel prognostic model for osteosarcoma using circulating CXCL10 and FLT3LG, Cancer 123 (2017) 144-154. 10.1002/cncr.30272.

7.Z.-L. Wu, Y.-J. Deng, G.-Z. Zhang, E.-H. Ren, W.-H. Yuan, Q.-Q. Xie, Development of a novel immunerelated genes prognostic signature for osteosarcoma, Sci Rep 10 (2020) 18402. 10.1038/s41598-02075573-w.

8.Y. Deng, W. Yuan, E. Ren, Z. Wu, G. Zhang, Q. Xie, A four-methylated LncRNA signature predicts survival of osteosarcoma patients based on machine learning, Genomics 113 (2021) 785-794. 10.1016/j.ygeno.2020.10.010.

9.K. Chen, N. Rajewsky, The evolution of gene regulation by transcription factors and microRNAs, Nat Rev Genet 8 (2007).

10.L.N. Heppler, D.A. Frank, Targeting Oncogenic Transcription Factors: Therapeutic Implications of Endogenous STAT Inhibitors, Trends Cancer 3 (2017) 816-827. 10.1016/j.trecan.2017.10.004.

11.A.S. Bhagwat, C.R. Vakoc, Targeting Transcription Factors in Cancer, Trends Cancer 1 (2015) 53-65.

12.C.M.J. Lucero, O.A. Vega, M.M. Osorio, J.C. Tapia, M. Antonelli, G.S. Stein, A.J. van Wijnen, M.A. Galindo, The cancer-related transcription factor Runx2 modulates cell proliferation in human osteosarcoma cell lines, J Cell Physiol 228 (2013) 714-723. 10.1002/jcp.24218.

13.F. Villanueva, H. Araya, P. Briceño, N. Varela, A. Stevenson, S. Jerez, F. Tempio, J. Chnaiderman, C. Perez, M. Villarroel, E. Concha, F. Khani, R. Thaler, F. Salazar-Onfray, G.S. Stein, A.J. van Wijnen, M. Galindo, The cancer-related transcription factor RUNX2 modulates expression and secretion of the matricellular protein osteopontin in osteosarcoma cells to promote adhesion to endothelial pulmonary cells and lung metastasis, J Cell Physiol 234 (2019) 13659-13679. 10.1002/jcp.28046.

14.G. Maurizi, N. Verma, A. Gadi, A. Mansukhani, C. Basilico, Sox2 is required for tumor development and cancer cell proliferation in osteosarcoma, Oncogene 37 (2018) 4626-4632. 10.1038/s41388-018-0292-2. 
15.M. Ruh, M.P. Stemmler, I. Frisch, K. Fuchs, R. van Roey, J. Kleemann, M. Roas, H. Schuhwerk, R.L. Eccles, A. Agaimy, D. Baumhoer, G. Berx, F. Müller, T. Brabletz, S. Brabletz, The EMT transcription factor ZEB1 blocks osteoblastic differentiation in bone development and osteosarcoma, J Pathol 254 (2021) 199-211. 10.1002/path.5659.

16.Y. Liu, S. Liao, S. Bennett, H. Tang, D. Song, D. Wood, X. Zhan, J. Xu, STAT3 and its targeting inhibitors in osteosarcoma, Cell Prolif 54 (2021) e12974. 10.1111/cpr.12974.

17.S.A. Lambert, A. Jolma, L.F. Campitelli, P.K. Das, Y. Yin, M. Albu, X. Chen, J. Taipale, T.R. Hughes, M.T. Weirauch, The Human Transcription Factors, Cell 172 (2018) 650-665. 10.1016/j.cell.2018.01.029.

18.K. Yoshihara, M. Shahmoradgoli, E. Martínez, R. Vegesna, H. Kim, W. Torres-Garcia, V. Treviño, H. Shen, P.W. Laird, D.A. Levine, S.L. Carter, G. Getz, K. Stemke-Hale, G.B. Mills, R.G.W. Verhaak, Inferring tumour purity and stromal and immune cell admixture from expression data, Nat Commun 4 (2013) 2612. 10.1038/ncomms3612.

19.P. Charoentong, F. Finotello, M. Angelova, C. Mayer, M. Efremova, D. Rieder, H. Hackl, Z. Trajanoski, Pan-cancer Immunogenomic Analyses Reveal Genotype-Immunophenotype Relationships and Predictors of Response to Checkpoint Blockade, Cell Rep 18 (2017) 248-262. 10.1016/j.celrep.2016.12.019.

20.J. Ritter, S.S. Bielack, Osteosarcoma, Ann Oncol 21 Suppl 7 (2010) vii320-vii325.

10.1093/annonc/mdq276.

21.J.C. Wittig, J. Bickels, D. Priebat, J. Jelinek, K. Kellar-Graney, B. Shmookler, M.M. Malawer, Osteosarcoma: a multidisciplinary approach to diagnosis and treatment, Am Fam Physician 65 (2002) 1123-1132.

22.Z.B. Abrams, M. Zucker, M. Wang, A. Asiaee Taheri, L.V. Abruzzo, K.R. Coombes, Thirty biologically interpretable clusters of transcription factors distinguish cancer type, BMC Genomics 19 (2018) 738. 10.1186/s12864-018-5093-z.

23.T.-H. Zhou, J.-Z. Su, R. Qin, X. Chen, G.-D. Ju, S. Miao, Prognostic and Predictive Value of a 15 Transcription Factors (TFs) Panel for Hepatocellular Carcinoma, Cancer Manag Res 12 (2020) 1234912361. 10.2147/CMAR.S279194.

24.B. Zhang, H. Wang, Z. Guo, X. Zhang, A panel of Transcription factors identified by data mining can predict the prognosis of head and neck squamous cell carcinoma, Cancer Cell Int 19 (2019) 297. 10.1186/s12935-019-1024-6.

25.Q. Liang, C. Xu, X. Chen, X. Li, C. Lu, P. Zhou, L. Yin, R. Qian, S. Chen, Z. Ling, N. Sun, The roles of Mesp family proteins: functional diversity and redundancy in differentiation of pluripotent stem cells and mammalian mesodermal development, Protein Cell 6 (2015) 553-561. 10.1007/s13238-015-0176-y. 
26.S. Chabab, F. Lescroart, S. Rulands, N. Mathiah, B.D. Simons, C. Blanpain, Uncovering the Number and Clonal Dynamics of Mesp1 Progenitors during Heart Morphogenesis, Cell Rep 14 (2016).

10.1016/j.celrep.2015.12.013.

27.N. Tandon, K. Goller, F. Wang, B. Soibam, M. Gagea, A.K. Jain, R.J. Schwartz, Y. Liu, Aberrant expression of embryonic mesendoderm factor MESP1 promotes tumorigenesis, EBioMedicine 50 (2019) 55-66. 10.1016/j.ebiom.2019.11.012.

28.S.S.-K. Chan, X. Shi, A. Toyama, R.W. Arpke, A. Dandapat, M. lacovino, J. Kang, G. Le, H.R. Hagen, D.J. Garry, M. Kyba, Mesp1 patterns mesoderm into cardiac, hematopoietic, or skeletal myogenic progenitors in a context-dependent manner, Cell Stem Cell 12 (2013) 587-601. 10.1016/j.stem.2013.03.004.

29.K. Yamazawa, T. Inoue, Y. Sakemi, T. Nakashima, H. Yamashita, K. Khono, H. Fujita, K. Enomoto, K. Nakabayashi, K. Hata, M. Nakashima, T. Matsunaga, A. Nakamura, K. Matsubara, T. Ogata, M. Kagami, Loss of imprinting of the human-specific imprinted gene causes prenatal growth retardation and dysmorphic features: implications for phenotypic overlap with Silver-Russell syndrome, J Med Genet 58 (2021) 427-432. 10.1136/jmedgenet-2020-107019.

30.R. Xu, S. Li, S. Guo, Q. Zhao, M.J. Abramson, S. Li, Y. Guo, Environmental temperature and human epigenetic modifications: A systematic review, Environ Pollut 259 (2020) 113840.

10.1016/j.envpol.2019.113840.

31.M. Kagami, K. Matsubara, K. Nakabayashi, A. Nakamura, S. Sano, K. Okamura, K. Hata, M. Fukami, T. Ogata, Genome-wide multilocus imprinting disturbance analysis in Temple syndrome and Kagami-Ogata syndrome, Genet Med 19 (2017) 476-482. 10.1038/gim.2016.123.

32.Y. Tanabe, A. Hirano, T. Iwasato, S. Itohara, K. Araki, T. Yamaguchi, T. Ichikawa, T. Kumanishi, Y. Aizawa, H. Takahashi, A. Kakita, H. Nawa, Molecular characterization and gene disruption of a novel zincfinger protein, HIT-4, expressed in rodent brain, J Neurochem 112 (2010) 1035-1044. 10.1111/j.14714159.2009.06525.x.

33.T. Inoue, H. Yagasaki, J. Nishioka, A. Nakamura, K. Matsubara, S. Narumi, K. Nakabayashi, K. Yamazawa, T. Fuke, A. Oka, T. Ogata, M. Fukami, M. Kagami, Molecular and clinical analyses of two patients with UPD(16)mat detected by screening 94 patients with Silver-Russell syndrome phenotype of unknown aetiology, J Med Genet 56 (2019) 413-418. 10.1136/jmedgenet-2018-105463.

34.N. Tarek, D.A. Lee, Natural killer cells for osteosarcoma, Adv Exp Med Biol 804 (2014) 341-353. 10.1007/978-3-319-04843-7_19.

35.L. Fernández, J. Valentín, M. Zalacain, W. Leung, A. Patiño-García, A. Pérez-Martínez, Activated and expanded natural killer cells target osteosarcoma tumor initiating cells in an NKG2D-NKG2DL dependent manner, Cancer Lett 368 (2015) 54-63. 10.1016/j.canlet.2015.07.042. 
36.C. Zhang, Y. Hu, C. Shi, Targeting Natural Killer Cells for Tumor Immunotherapy, Front Immunol 11 (2020) 60. 10.3389/fimmu.2020.00060.

37.E.P. Buddingh, M.W. Schilham, S.E.N. Ruslan, D. Berghuis, K. Szuhai, J. Suurmond, A.H.M. Taminiau, H. Gelderblom, R.M. Egeler, M. Serra, P.C.W. Hogendoorn, A.C. Lankester, Chemotherapy-resistant osteosarcoma is highly susceptible to IL-15-activated allogeneic and autologous NK cells, Cancer Immunol Immunother 60 (2011) 575-586. 10.1007/s00262-010-0965-3.

38.X. Yang, W. Zhang, P. Xu, NK cell and macrophages confer prognosis and reflect immune status in osteosarcoma, J Cell Biochem (2018). 10.1002/jcb.28167.

\section{Figures}

A

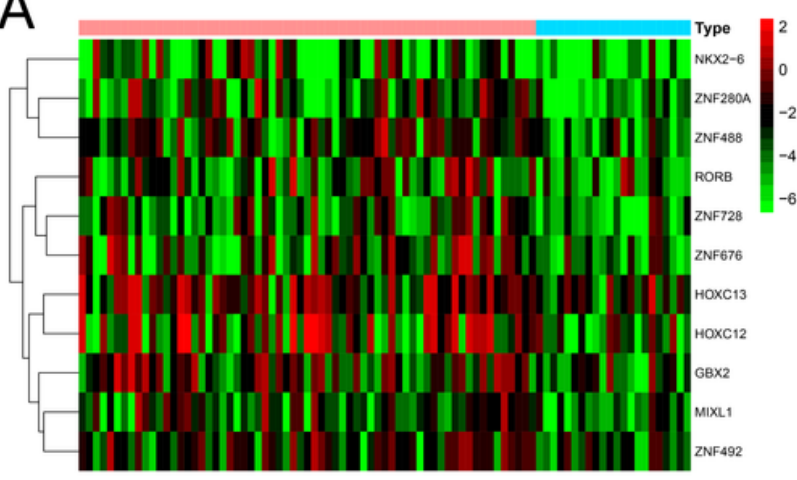

B Type 白 Metastatic 白 Non-metastatic

Metastatic

Non-metastatic

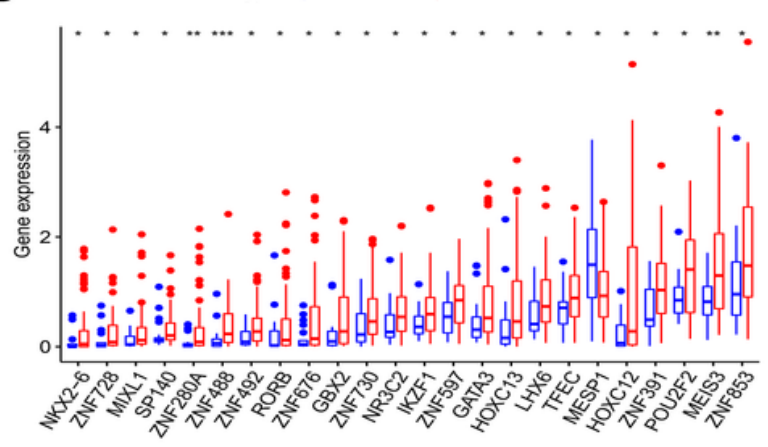

C

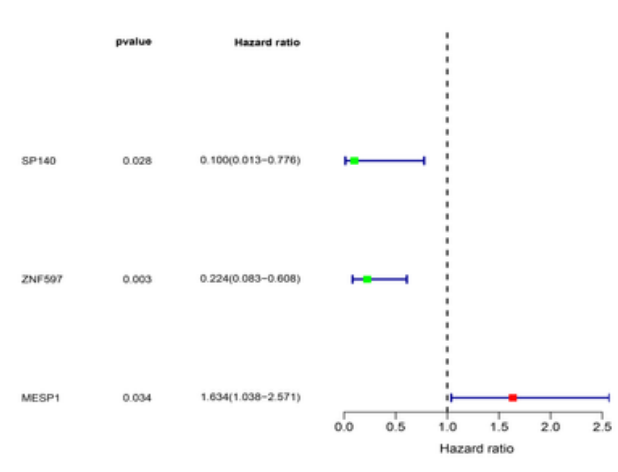

E

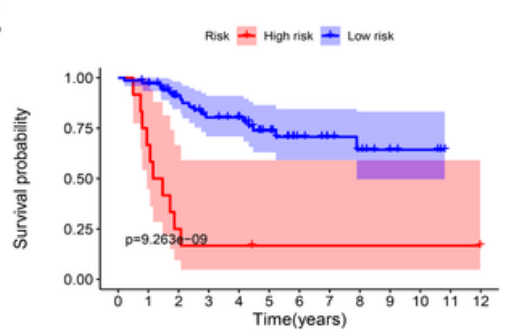

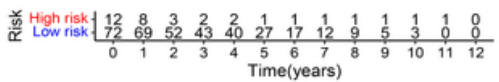

$\mathrm{F}$

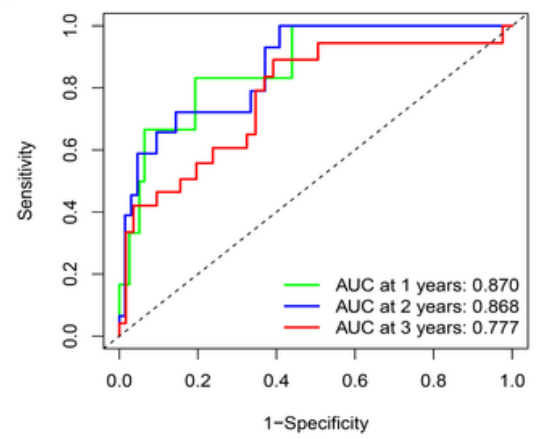

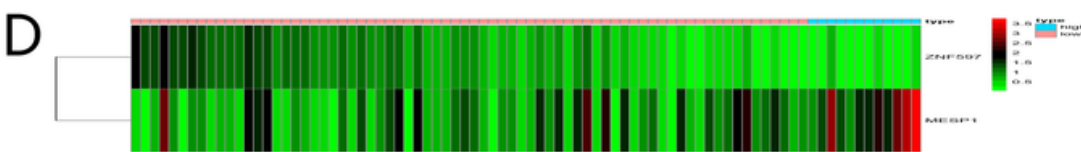
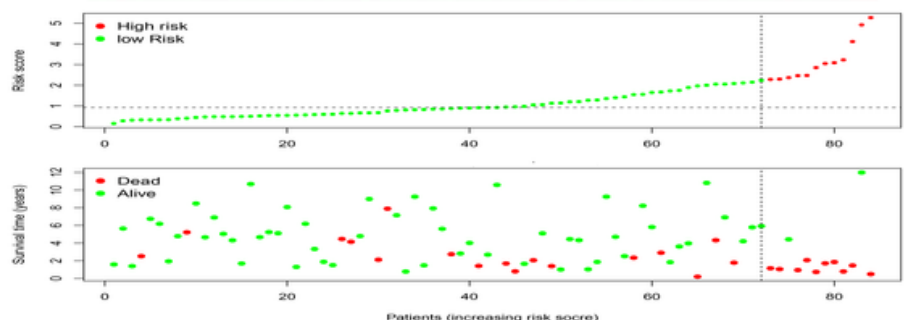

G

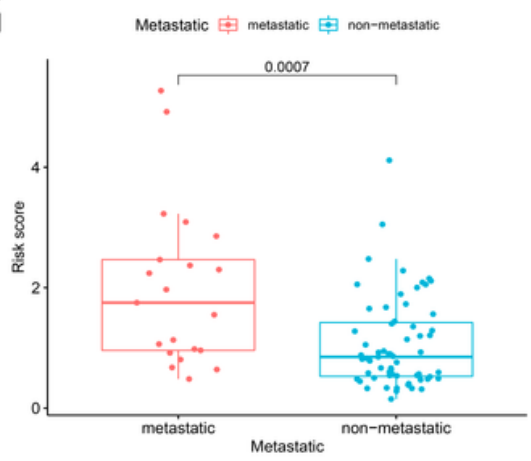


Figure 1

Construction and assessment of the TFs-related prognostic signature. Heatmap (A) and box plot (B) of the DETFs between metastatic and non-metastatic osteosarcoma samples from the TARGET database. Red represents higher expression genes, green represents lower expression genes, black represents the same expression genes. (C) Forest plot of univariate Cox regression analysis of the survival-related 3 DETFs in osteosarcoma. (D) The risk scores, survival status, and gene expression levels between the high-risk and low-risk groups. Kaplan-Meier survival analysis (E) and ROC analysis of the risk scores for 1,2 , and 3 years overall survival prediction $(F)$. (G) Risk scores between metastasis and non-metastasis groups. (TARGET: Therapeutically Applicable Research to Generate Effective Treatments; DETFs: differentially expressed transcription factors; ROC: receiver operating characteristic)
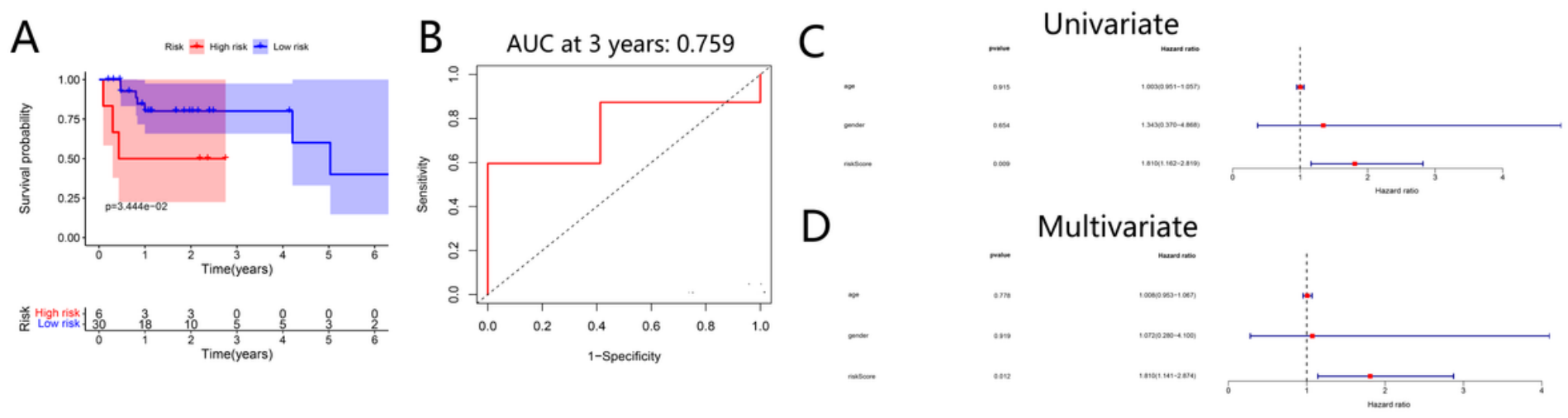

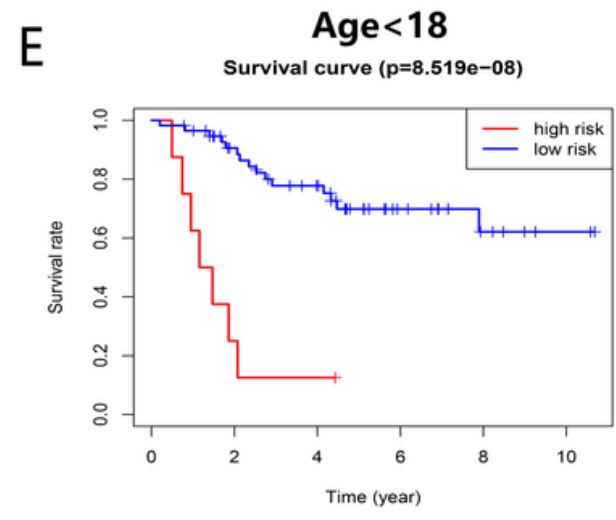

Age $>=18$

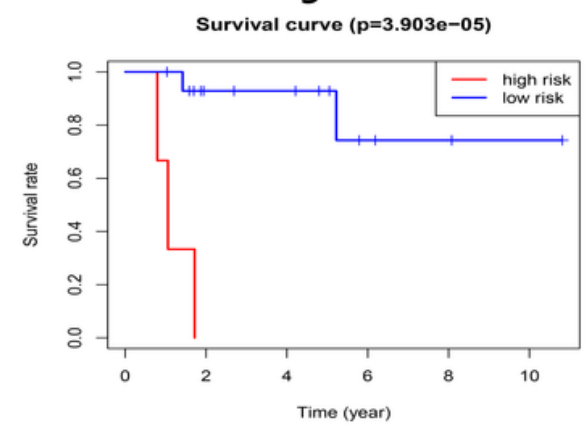

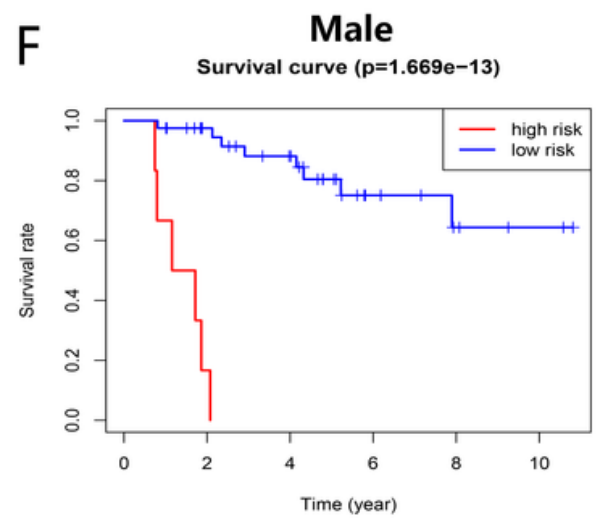

Female

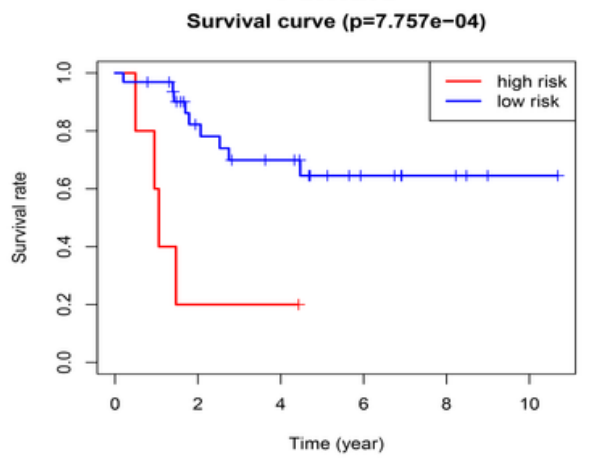

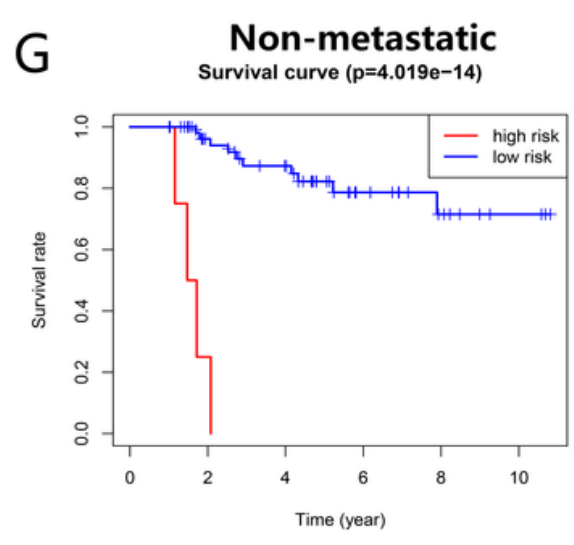

Metastatic

Survival curve $(p=4.06 e-02)$

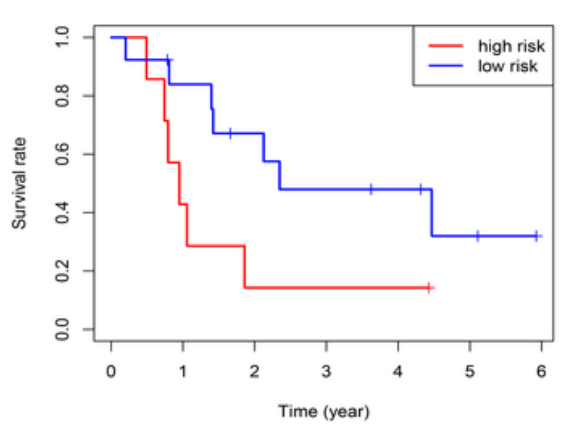

Figure 2 
Validation of the TFs-related prognostic signature in GSE39055 and multiple clinical subtypes analysis. (A) Kaplan-Meier survival plot. (B) Survival prediction of the risk signature was assessed by ROC curve for 3 years. (C,D) Univariate and multivariate Cox regression analysis of risk score and different clinical features in osteosarcoma. Kaplan-Meier curve of osteosarcoma patients (E)age $<18$ years and age $\geq 18$ years; (F)male and female, and (G)metastatic and non-metastatic in the TARGET database.

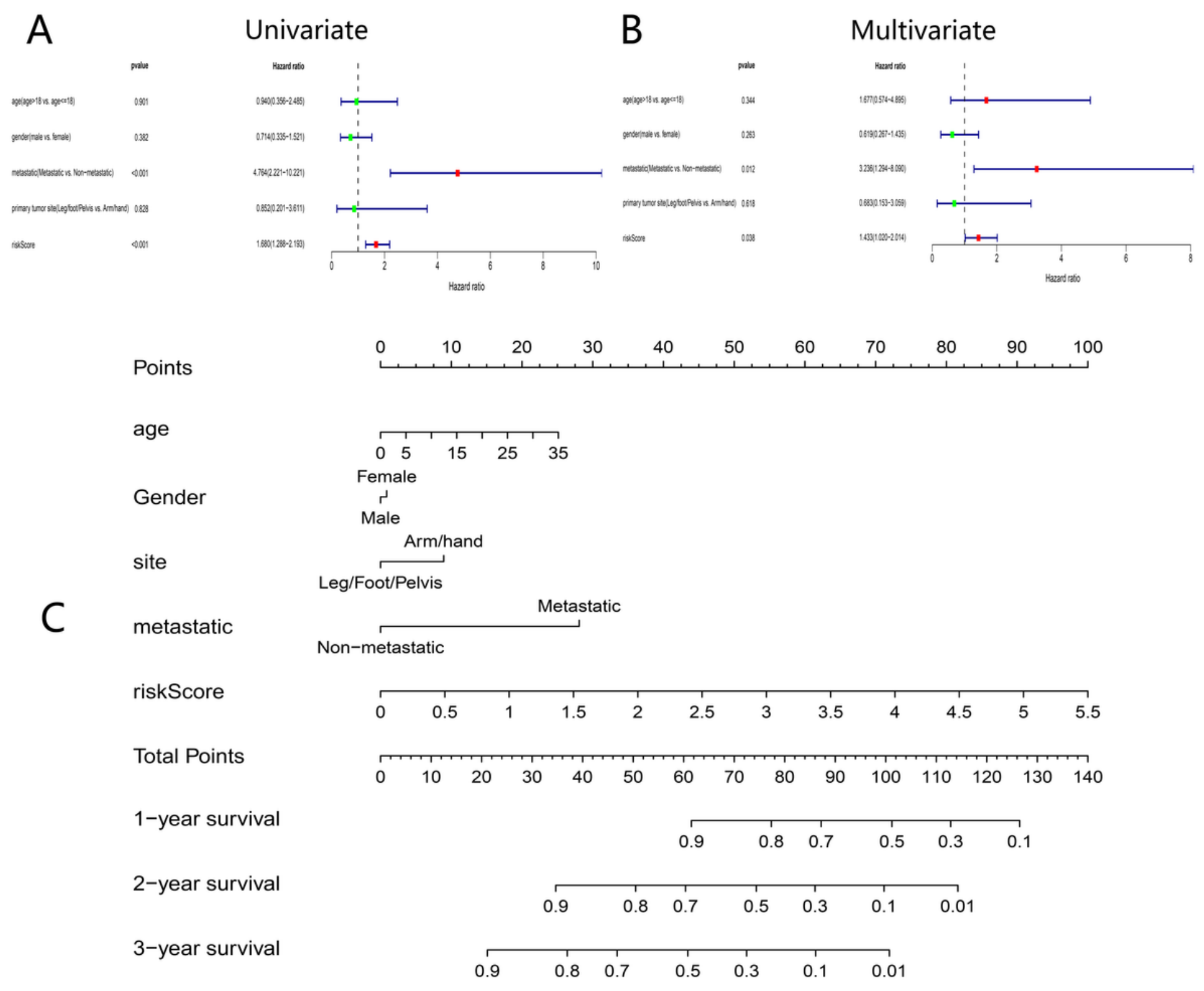

Figure 3

Prognostic capacity evaluation and nomogram analysis of the TFs-related prognostic signature in the TARGET database. (A) Univariate cox regression analysis of risk score and different clinical features in osteosarcoma. (B) Multivariate cox regression analysis of risk score and different clinical features in osteosarcoma. (C) The nomogram to predict the 3- and 5-year survival risk of osteosarcoma patients. 
A

StromalScore $(p=0.02)$

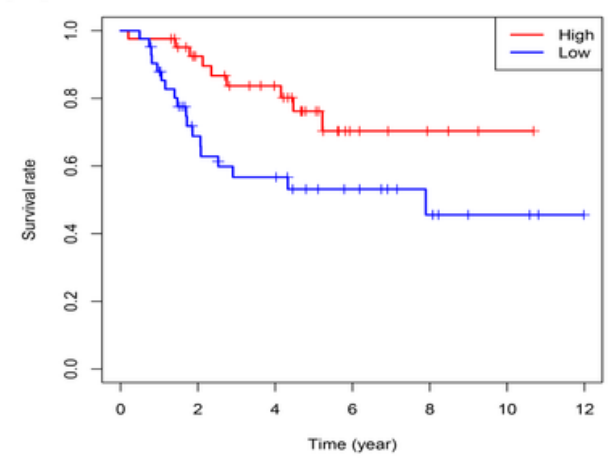

D

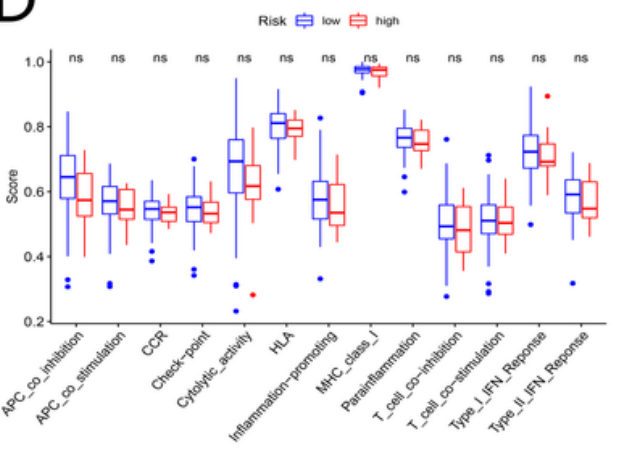

$\mathrm{E}$

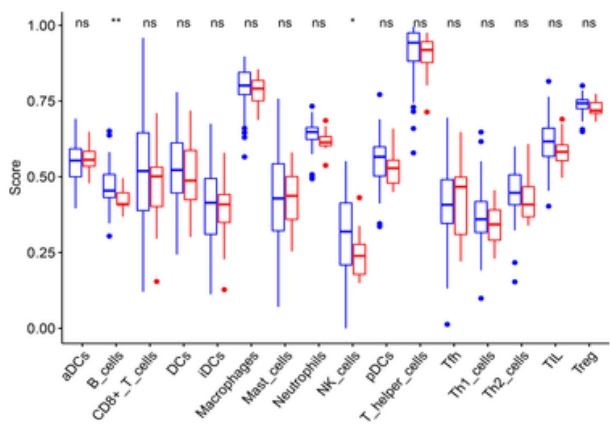

B

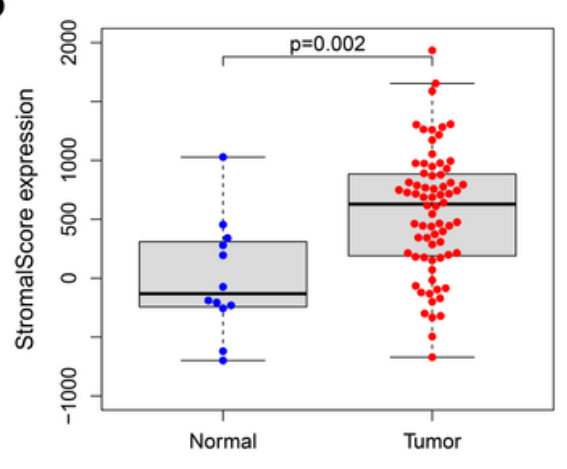

F

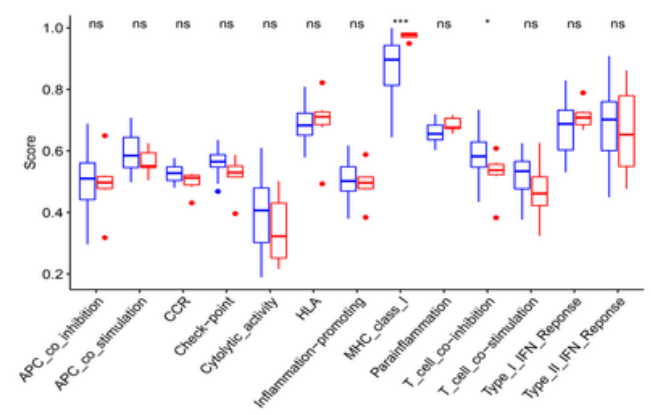

G

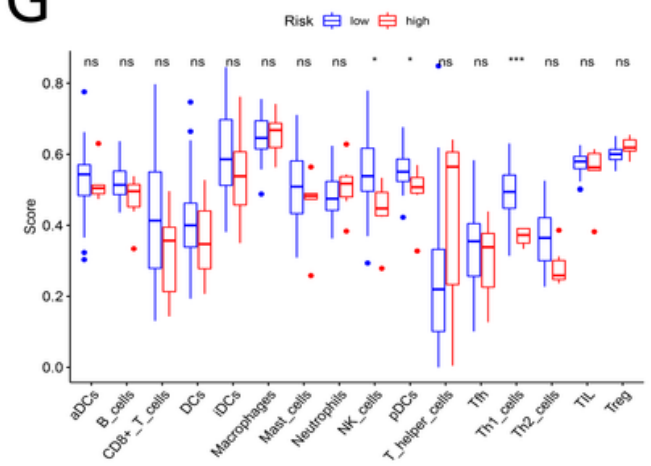

C
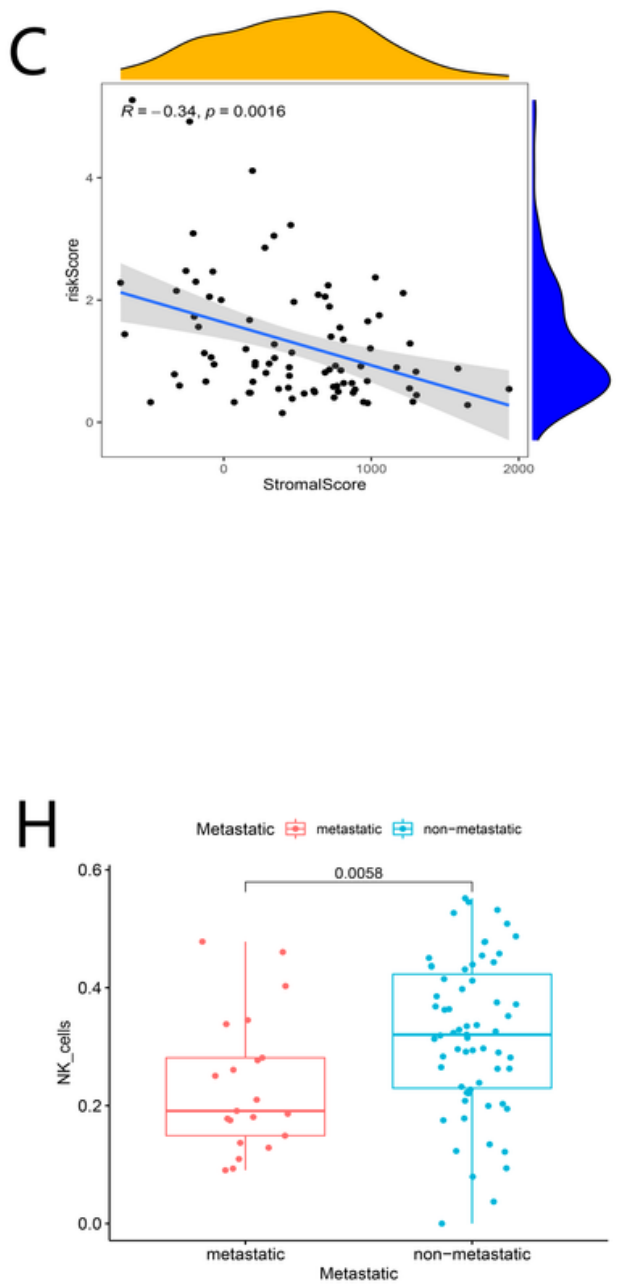

Figure 4

The difference of tumor environment between high- and low-risk groups in the TARGET database. (A) Kaplan-Meier survival curve of stromal score among the high-risk and low-risk group (B) The difference of stromal score between the high-risk prognostic and low-risk prognostic group. (C) The correlation analysis between the risk score and the stromal score. (D, E) Differences in 16 immune cells and 13 immune characteristics were found in the TARGET database between the high- and low-risk prognostic groups. $(F, G)$ The correlations between risk score and different immune cells and immune features in the GSE39055 dataset. (H) The abundance of NK cells between metastasis and non-metastasis groups.

\section{Supplementary Files}

This is a list of supplementary files associated with this preprint. Click to download. 
- SupplementTable2.xlsx

- SupplementaryFigure1.tif

- SupplementaryFigure2.tif

- SupplementaryFigure3.tif

- SupplementaryTable1.docx 